\title{
Vom Trugschluss, dass «mehr Markt im Gesundheitswesen» es richten könne
}

\author{
Diese Stellungnahme zum Artikel «Gesund, unpässlich, unwohl, krank - invalid: Spiel \\ ohne Grenzen?» von Constantin Schuler [1] plädiert für mehr Unterstützung bei der \\ beruflichen Wiedereingliederung psychisch beeinträchtigter Personen. Dies helfe \\ eher Folgekosten zu vermeiden als Konkurrenz auf einem «Gesundheitsmarkt».
}

Werner Saameli

Ehrenmitglied der Schweizerischen Gesellschaft für Sozialpsychiatrie

\section{Konkurrenz belebt das Geschäft, womit der Gesamt- umsatz, also das Total der Gesundheitskosten, steigt}

Korrespondenz: Dr. med. Werner Saameli Obere Hauptgasse 47 CH-3600 Thun

wrsaameli@gmx.ch
Die Verdienste des Kollegen Schuler um den Ausbau der medizinischen Abklärungsstellen der IV sind ebenso unbestritten wie sein Anliegen, aktuelle Probleme der IV-Finanzierung im Zusammenhang mit dem Wandel des Gesundheitsbegriffes anzugehen.

Wie im treffenden Kommentar von Kollege Hans Kurt [2] ausgeführt, ist aus sozialpsychiatrischer Sicht allerdings Widerspruch gegen die abschätzig undifferenzierte, eigentlich als überholt geltende Diskreditierung dieses Fachbereiches zu erheben. Es waren nämlich präzis die Vertreter der Sozialpsychiatrie, die sich schon vor Jahrzehnten mit Erfolg für die berufliche Wiedereingliederung von psychisch Beeinträchtigten einsetzten. Die Ressource, an einem geschützten (institutionellen) oder einem beschützenden Arbeitsplatz in der freien Wirtschaft im Sinne des «supported employment» gesellschaftlich integriert zu bleiben, wird gerade von diesem Fachbereich sehr ausgiebig (und öfters auch rentenwirksam) zugunsten der Krankheitsbewältigung durch die Betroffenen genutzt. schieben. Diese sind insbesondere in den Bereich der Ökonomisierung des Gesundheitswesens einzuordnen, an dem noch ganz andere Akteure als die Ärztinnen und Ärzte beteiligt sind.

\section{Als Kunde will man für seine teure Versicherung möglichst viel bekommen}

Die Steuerung des Gesundheitswesens hat sich in den letzten Jahrzehnten von einer vorwiegend normativen zu einer überwiegend pekuniären entwickelt. Anstelle von Forderungen zur Optimierung (z.B. der Zugänglichkeit oder Betreuungsqualität) steht nun der Ruf nach mehr Markt im Gesundheitswesen im Vordergrund. Dies in der Hoffnung, dass durch die Konkurrenz der Leistungserbringer bzw. der Krankenversicherer untereinander die Preise gesenkt werden. Dies ist aber ein Trugschluss: Wohl kann der Konkurrenzkampf die Preise einzelner Produkte drücken und damit verbilligen, doch ist andererseits unbestritten erwiesen, dass «die Konkurrenz das Geschäft belebt», womit der Gesamtumsatz, also das Total der Gesundheitskosten, steigt. Nicht umsonst erwartet man ja von den Märkten ständiges quantitatives Wachstum, um die Wirtschaft gesund zu erhalten. Die qualitativen Verbesserungen sind durch den kompetitiven akademischen Anspruch innerhalb einer handlungswissenschaftlichen Disziplin ja von vorneherein gegeben. Da die Gesundheitskosten mehrheitlich von der Solidargemeinschaft (Krankenkassen, Versicherungen, Steuern) bezahlt werden, wirkt sich die durch Konkurrenz provozierte Mengenausweitung als volkswirtschaftlich relevante Gesamtkostensteigerung aus. Dass nun gar die ehemals als Patienten bezeichneten Kranken als Kunden bezeichnet werden, entlarvt die leistungserbringenden Unternehmungen erst recht als Verkäufer, die nun willige Konsumenten vor sich haben. 
Schon 1976 hatte Ivan Illich [4] kommen sehen, dass der Gesundheitsmarkt die Nachfrage nach Medikamenten und nach medizinischen Leistungen schürt und die öffentlichen Ressourcen über das vertretbare Mass hinaus absorbiert.

Die (auf dem Hintergrund des als Ersatzreligion funktionierenden Ökonomismus) um sich greifende Ökonomisierung aller Lebensbereiche inklusive Gesundheitswesen ist ein wichtiger Faktor der zunehmenden Medikalisierung. Aus lauter Befürchtung, die Kranken würden als wehrlose passive Patienten von überaktiven Ärzten und ihrer Medizintechnik als Objekte missbraucht, hat sich eine starke Tendenz durchgesetzt, sie zu autonomen und mündigen Konsumenten von angebotenen medizinischen Leistungen zu machen. Sie können selber entscheiden, was sie wollen. Gemeint war, dass sie die von Ärzten vorgeschlagenen Massnahmen ablehnen können. Da sie für die Versicherung aber viel zahlen, verhalten sie sich durchaus auch als Kunden, die möglichst viel dafür wollen. Sie entscheiden sich nun autonom (und

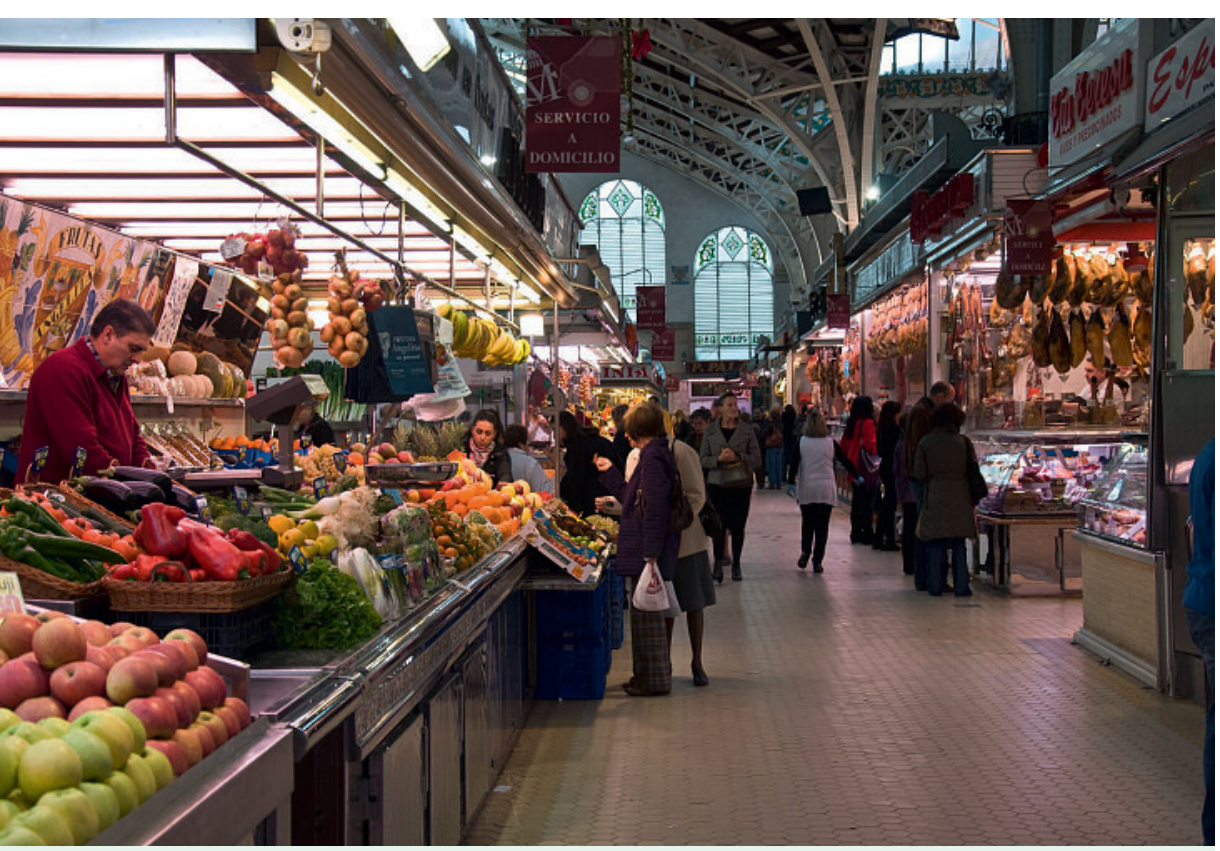

Konkurrenz belebt das Geschäft - doch ein grösseres Angebot weckt auch eine grössere Nachfrage. manchmal gierig) für das, was auf dem Markt angeboten wird. Im gewünschten Markt verkaufen medizinische Einrichtungen und die Industrie gerne, was gewünscht wird; marktkonform schaffen sie auch neue Bedürfnisse. Dabei geht es schon weniger um Linderung von Leiden als um ein «nice to have» und um «human enhancement» [5]. Man hat die passiv Leidenden zu aktiven Partnern in einem Markt werden lassen und kritisiert die diesen ausweitenden Pharmafirmen und die Lifestyle-Praxen, die hochqualifiziertes Medizinalpersonal aus der Versorgung von im herkömmlichen Sinn Kranken abziehen.

Darum vertrete ich die These, dass weniger Markt im Gesundheitswesen die Medikalisierung eindämmen würde. Stattdessen wäre eine wissenschaftlich begründete Unterstützung der kranken Subjekte im Sinne des Empowerments zur Bewältigung ihrer Krankheitszustände die empfehlenswerte Strategie der Zukunft. Dies, um die Gesundheitskosten und die Ausweitung des Krankheitsbegriffes und eventuell (laut Schuler) auch die Inflation von Ansprüchen, als invalid zu gelten, in den Griff zu bekommen. Im Klartext: Mehr Schulung und weniger Markt im Gesundheitswesen! Mehr Unterstützung zur Verhinderung von Arbeitsplatzverlusten bei gesundheitlich bedingten Leistungseinbussen statt unendliche Wiederholungen von Abklärungen bis zur Produktion von (Pseudo-?) Objektivität. Anstelle des Ausschlusses von Sozialfaktoren Nutzung derselben zur Optimierung des Behandlungsprozesses.

\section{Literatur}

1 Schuler C. Gesund, unpässlich, unwohl, krank invalid: Spiel ohne Grenzen? Schweiz Ärztezeitung. 2010; 91(34):1299-1302.

2 Kurt H. Befindlichkeitsstörung oder psychiatrische Krankheit? Schweiz Ärztezeitung. 2010;91(34):1303.

3 Meyer PC. Medikalisierung als sozialer Prozess. SUZ. Vorlesung FS 2009.

4 Illich I. Die Nemesis der Medizin: die Kritik der Medikalisierung des Lebens. München: Beck, ergänzte Aufl.; 1995.

5 NEK- und SAMW-Symposium: Medizin für wen? Gesundheitsversorgung im Spannungsfeld zwischen den Bedürfnissen der Patienten und der Dynamik einer marktorientierten Medizin. 11. Juni 2010 